\title{
Differentiation between two strains of microalga Parachlorella kessleri using modern spectroscopic method
}

\author{
Marwa M Saleh ${ }^{1,3^{*}}$, Dmitry N Matorin², Bolatkhan K Zayadan', Daria A Todorenko², Evgenii P Lukashov² \\ and Mona M Gaballah ${ }^{3}$
}

\begin{abstract}
Background: The differentiation between wild type of Parachlorella kessleri and its mutant strains PC Mut2, PC Mut4 by using the Multi-functional Plant Efficiency Analyzer (M-PEA-2) was studied. Mutant algal cells of $P$. kessleri have been obtained by UV-C during 3 and 10 min respectively.

Results: Light-induced kinetics of prompt fluorescence (OJIP transients), delayed fluorescence and modulated reflection at $820 \mathrm{~nm}$ (redox transitions of $\mathrm{P}_{700}$ in PSI) showed disturbance of electron transport flow in photosystem II (PSII) and an increase fraction of non-reducing centers of secondary quinone acceptors of electron $\left(\mathrm{Q}_{\mathrm{B}}\right)$. In addition, the amplitudes of the fast and slow peak in the kinetics of the delayed light emission and non-photochemical fluorescence quenching (NPQ) were significantly reduced in mutant cells, indicating low level of the membrane energization of photosynthetic membranes. Changes of photosynthetic reactions of mutants may lead to an increase of the carotenoids content, which protect cells against the light stress.
\end{abstract}

Conclusion: It is suggested to use parameters of induction curves of prompt and delayed fluorescence to characterize mutant algal cells in biotechnological studies.

Keywords: Parachlorella kessleri; Chlorophyll a fluorescence; Delayed fluorescence; JIP-test; Photosynthesis

\section{Background}

The solar radiation sustains life on Earth. However significant increase of UV-C radiation may lead to inhibition of many biological processes. The major cellular targets of UV-C are various biomolecules, which directly absorb this radiation or are indirectly affected by various UV-C induced photochemical reactions. The biological and, ultimately, ecological consequences are numerous (Charles 2008). It is known that UV-C induces some physiological effects such as declining photosynthetic rates, which can be related not only to damaged biomolecules, but also to ultrastructural changes in organelles or membranes (Holzinger and Lütz 2006). Typical alterations include swollen mitochondrial cristae, disrupted thylakoids or

\footnotetext{
* Correspondence: marwasaleh2000@yahoo.com

${ }^{1}$ Department of Biotechnology, Al-Farabi Kazakh National University, 71,

Al-Farabi, ave, Almaty 050040, Kazakhstan

${ }^{3}$ Botany Department, Faculty of Science, Suez Canal University, Ismaillia

41522, Egypt

Full list of author information is available at the end of the article
}

detached phycobilisomes in chloroplasts, bent-shaped dictyosomes, and damaged plasmalemma. An intact ultrastructure of the algal cell is a prerequisite for the optimum functioning of all physiological processes (Charles 2008). Parachlorella kessleri was described as a new genus, derived from the true spherical Chlorella. The wild strain SAG 211-11 h was formerly referred to as Chlorella vulgaris but later reassigned as Chlorella kessleri (Fott and Nováková 1969). Today this species is referred to as $P$. kessleri (Krienitz et al. 2004).

Fluorescence methods are used in biotechnological experiments for monitoring photosynthesis processes, which give detailed information of primary defects of cell metabolism, mainly at the membrane level (Schreiber 2004; Antal et al. 2009; Matorin et al. 2013; Schansker et al. 2014). The basis of fluorescence methods lies in the ability of chlorophyll located in photosynthesis membranes to serve as a natural detector for the algae cells, emitting quanta fluorescence. Importantly, such 
methods allow to gain information of alga state in real time. Measurement of the ratio between the fluorescence intensity under the photosynthesis saturating illumination $\left(F_{M}\right)$ and under low light intensity, which induces no changes in the state of the photosynthetic apparatus $\left(F_{O}\right)$ makes it possible to determine the maximum efficiency of the PSII processes, which is equal to $F_{V} / F_{M=}\left(F_{M}-F_{O}\right) / F_{M}$. The $F_{V} / F_{M}$ ratio presents dimensionless characteristics of the efficiency of photosynthesis, which is independent of the species of organisms. Measurements of fluorescence induction curves are carried out during several seconds by using PAM or PEA instrument. Measurements of fluorescence induction curves with high resolution (starting from $20 \mu \mathrm{s}$ ) have been recently used in studies of photosynthetic reactions in higher plants and algae cultures (Schreiber 2004; Strasser et al. 2005; Matorin and Rubin 2012). The M-PEA-2 instrument allows to measure separate reactions in PSI and PSII simultaneously (Strasser et al. 2010; Oukarroum et al. 2013; Bulychev et al. 2013). Moreover, it monitors induction changes of delayed fluorescence, which indicate the level of the membrane energization (Goltsev et al. 2009).

In the present paper we investigated processes in PSI and PSII of wild type of P. kessleri and two mutant strains by using a newly designed Multi-functional Plant Efficiency Analyzer (M-PEA-2, Hansatech). This instrument gives a comprehensive picture of primary photosynthetic events by simultaneous recording the prompt and delayed fluorescence kinetics and reflectance at $820 \mathrm{~nm}$ at high time resolution. Here we introduce results of the first attempt of assessment of photosynthetic characteristics of the mutant strains of algal cells by using M-PEA-2.

\section{Methods}

\section{Strains and growth conditions}

The strain of Parachlorella kessleri microalga was obtained from Al-Farabi Kazakh National University, Biotechnology Department culture collection. P. kessleri wild type strain was grown in tris-acetate-phosphate (TAP) medium (pH 6-7) (Gorman 1965), in $250 \mathrm{ml}$ Erlenmeyer flask at $28^{\circ} \mathrm{C}$ under continuous illumination of photosynthetic photon flux density (PPFD) $120 \mu \mathrm{mol}$ photons $\mathrm{m}^{-2} \mathrm{~s}^{-1}$ and constant shaking.

\section{UV irradiation and mutagenesis}

According to Harris (1989), $5 \mathrm{ml}$ of the liquid culture with a density of $1 \times 10^{6} / \mathrm{ml}$ algal cells were placed in $9 \mathrm{~cm}$ Petri dish, where the culture formed a thin layer, covering the bottom. The dish was placed on shaker at $20 \mathrm{rpm}$ and exposed to UV-C lamp (254 $\mathrm{nm}$ and $40 \mathrm{erg} / \mathrm{mm}^{2}$ ) at distance $15 \mathrm{~cm}$ during 3 and $10 \mathrm{~min}$ respectively. After UV irradiation, the cells were inoculated in solid TAP medium and incubated in darkness for $24 \mathrm{~h}$ to prevent photoreactivation. After the $24 \mathrm{~h}$, some dishes were incubated photoautotrophically (photon flux density $120 \mu \mathrm{mol}$ photons $\mathrm{m}^{-2} \mathrm{~s}^{-1}$ ), others were incubated heterotrophically (in dark) during 15 days.

\section{Selection of the mutant strains}

After the incubation period, two mutant strains were selected based on their phenotypic characteristics, using colonies whose color and size differed from the wild type colonies. These mutant strains, PC Mut2 and PC Mut4, were obtained from $P$. kessleri after irradiation time of 3 and $10 \mathrm{~min}$ respectively. These mutants were transferred from the solid to liquid medium and kept under phototrophic growth conditions (photon flux density $120 \mu \mathrm{mol}$ photons $\mathrm{m}^{-2} \mathrm{~s}^{-1}$ ).

\section{Analysis of chlorophyll $a$ and $b$ content}

Spectrophotometry method was used according to Merchant et al. (2007). The calculation of the concentration of the pigments was determined by the optical density of pigment solutions at appropriate wavelength.

\section{Chlorophyll fluorescence and absorbance measurements}

Light-induced kinetics of prompt Chl fluorescence (PF), delayed Chl fluorescence (DF) and modulated reflectance at $820 \mathrm{~nm}$ (MR) were recorded by using Multi-functional Plant Efficiency Analyzer (M-PEA-2, Hansatech Instrument Ltd., King's Lynn, Norfolk, UK). Prior to the measurements, the cells were concentrated on a membrane filter. Then a thin layer of cells was fixed by a special clip on the surface of the measuring chamber and incubated in the darkness for $10 \mathrm{~min}$. It has previously been verified in algal suspension by using Aqua-Pen fluorometer (Photon System Instruments, Czech Republic) that concentration algal cells on the filter surface doesn't affect the physiological state of cells (Matorin et al. 2013).

The design of the measuring chamber, timing protocol of a single measurement, and data acquisition procedure for M-PEA-2 can be found in Strasser et al. (2010). PPFD and duration of actinic illumination were set to $1300 \mu \mathrm{mol}$ photons $\mathrm{m}^{-2} \mathrm{~s}^{-1}$ and $3 \mathrm{~s}$, respectively. In the M-PEA-2, the DF induction curves are constructed from the DF intensities at certain delay time-point in the kinetics of DF decay recorded during the dark interruptions of the actinic light, which induces OJIP (intermediate levels of the light-induced fluorescence curve) rise. In our experiments, the delay time point in the DF decay curve was set to $50 \mu \mathrm{s}$. It is assumed that submillisecond DF components are generated from PSII reaction centers in the state $\mathrm{Z}^{+} \mathrm{P}_{680} \mathrm{Q}_{\mathrm{A}}^{-}$(Goltsev et al. 2009), where $\mathrm{Z}$ is a tyrosine $\mathrm{Z}$ residue, $\mathrm{P}_{680}$ is a primary electron donor, and $\mathrm{Q}_{\mathrm{A}}$ is a primary quinone acceptor in PSII.

Fluorescence was measured by using Water-PAM fluorometer (Heiz Walz, Effeltrich, Germany). Ratio $F_{V} / F_{M}$ is the maximum quantum yield of PSII photochemistry was 
measured in dark-adapted sample, where $F_{V}=F_{M}-F_{O}$. Measurements in the light were performed with progressive increase of light intensity from 0 to $800 \mu \mathrm{mol}$ photons $\mathrm{m}^{-2} \mathrm{~s}^{-1}$. At the end of each illumination session, $F_{M}{ }^{\prime}$ and fluorescence yield in light $F(t)$ were measured using saturating flash $\left(0.8 \mathrm{~s}, 3000 \mu \mathrm{mol}\right.$ photons $\left.\mathrm{m}^{-2} \mathrm{~s}^{-1}\right)$ (Schreiber 2004; Herlory et al. 2007). These parameters were used to calculate non-photochemical quenching of chlorophyll fluorescence $N P Q=\left(F_{M}-F_{M}{ }^{\prime}\right) / F_{M}{ }^{\prime}$, the quantum yield of photochemical conversion of light energy in PSII, as $Y=\left(F_{M^{\prime}}-F(t)\right) / F_{M}^{\prime}$, and the relative yield of noncyclic electron transport at given light intensity $r E T R=Y \times E_{i} \times 0.5$, where $E_{i}$ is illumination intensity $\left(\mu \mathrm{mol}\right.$ photons $\mathrm{m}^{-2} \mathrm{~s}^{-1}$ ) (Lippemeier et al. 1999). Parameters are designated according to generally accepted nomenclature (Schreiber 2004).

Light absorption spectrum of samples was measured by using HITACHI-557 spectrophotometer. Fluorescence spectra, excitation spectra, and fluorescence decay times in algae were measured by using Fluorolog-3 (Horiba Jobin Yvon) spectrofluorometer with the TCSPC (time correlated single photon counting) option (O'Connor and Philips 1984). Fluorescence was measured at $685 \mathrm{~nm}$ under excitation with pulse LED at $390 \mathrm{~nm}$. Fluorescence decay kinetics was approximated with the sum of two exponential curves.

All measurements were repeated no less than five times. The figures present data of three replicate experiments at least.

\section{Results and discussion}

In this study mutations were induced by UV-C radiation. UV-C mutagenesis offers many advantages such as less pollution, simple operation, and sterile cultivation conditions. After UV-C irradiation, some colonies appeared with phenotypic characteristics that differed in color and size from the control colonies. The colonies were selected based on their phenotypic appearance, transferred into liquid medium and then subcloned several times to insure that the mutation was stable and no changes in their phenotypic characteristics occurred. The major cellular targets of UV-C are different biomolecules, which directly absorb this radiation, or which are indirectly affected by various UV-C induced photochemical reactions (Charles 2008). In the present paper two mutant strains of $P$. kessleri after irradiation during 3 and 10 min respectively were selected and designated as $P C M u t 2$ and $P C$ Mut4. It was shown microscopically that mutant cells are smaller, than wild type cells. All strains were grown in the liquid TAP medium under phototrophic conditions. The algal cultures of the wild and mutant strains were different in color.

Absorption spectra of wild type and mutant strains of cells are in Figure 1. The spectra have been normalized

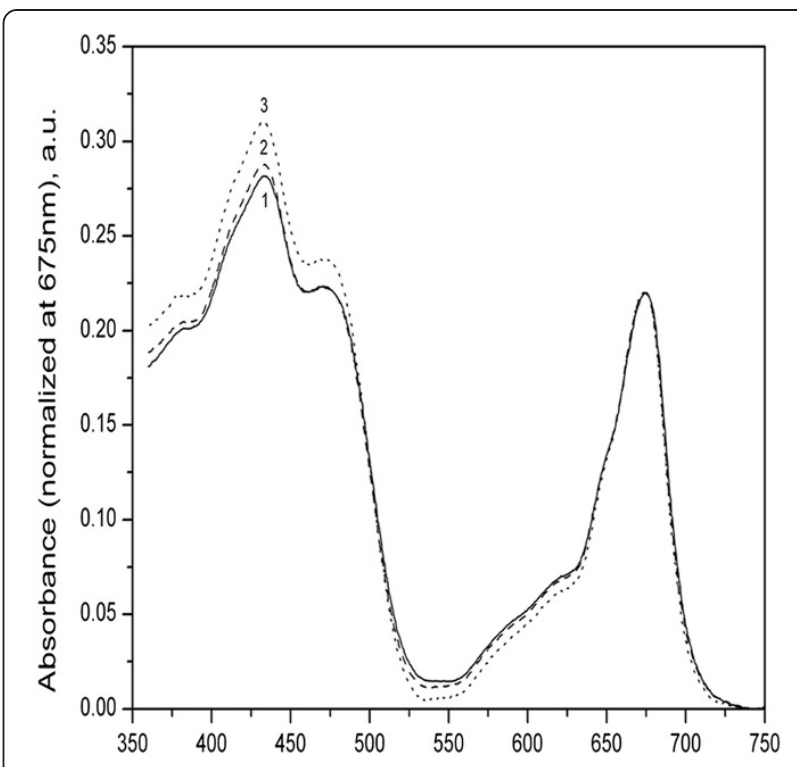

Figure 1 Absorption spectra of suspensions. wildtype (curve 1) and mutant strains PCMut2 (curve 2) and PCMut4 (curve 4). The spectra are normalized to absorption in the red maximum of chlorophylla $a$ at $675 \mathrm{~nm}$.

to the maximum chlorophyll absorption in red region at $675 \mathrm{~nm}$. Relative increase of carotenoid absorption at the Soret band was noted in mutant strains of $P$. kessleri. The highest increase was observed in PC Mut4. This was confirmed by measurements of fluorescence excitation spectra (Figure 2B) The se spectra showed an increase in the region of carotenoid absorption. Fluorescence spectra did not differ significantly (Figure 2A), which may be related to the fact that carotenoids in algae do not fluorescence, but efficiently transfer excitation energy of chlorophyll $a$, which is fluorescence emitter.

It is known that the constant fluorescence level $F_{O}$ highly correlates with the total content of pigments in the photosynthetic apparatus of algal cells, that harvest light energy, and $F_{O}$ level correlates with cell number (Matorin et al. 2004). The ratio $F_{V} / F_{M}$ reflects the maximum quantum yield in PSII. This value is related to the processes of water splitting and oxygen evolution in PSII. Changes of fluorescence parameters $\left(F_{O}, F_{V} / F_{M}\right)$ are shown in Figure 3.

Figure 3 shows fast increase of algal cells of wild type (curve 1$)$ after the lag-phase ( $1^{\text {st }}$ day), as measured by $F_{O}$. PC Mut2 (curve 2) and PC Mut4 (curve 3) mutants demonstrate a lower growth rate during investigation period. Photosynthetic activity $\left(F_{V} / F_{M}\right.$ ratio) of wild type cells slightly increased during growth, reaching the maximum value at the $3^{\text {rd }}$ day. After prolonged culturing $\left(6^{\text {th }}\right.$ day) photosynthetic activity, judged by $F_{V} / F_{M}$ ratio, was decreased, possibly due to exhaustion of mineral nutrients in the growth medium. Mutants $P C$ Mut2 and $P C$ Mut 4 had a lower $F_{V} / F_{M}$ values. 

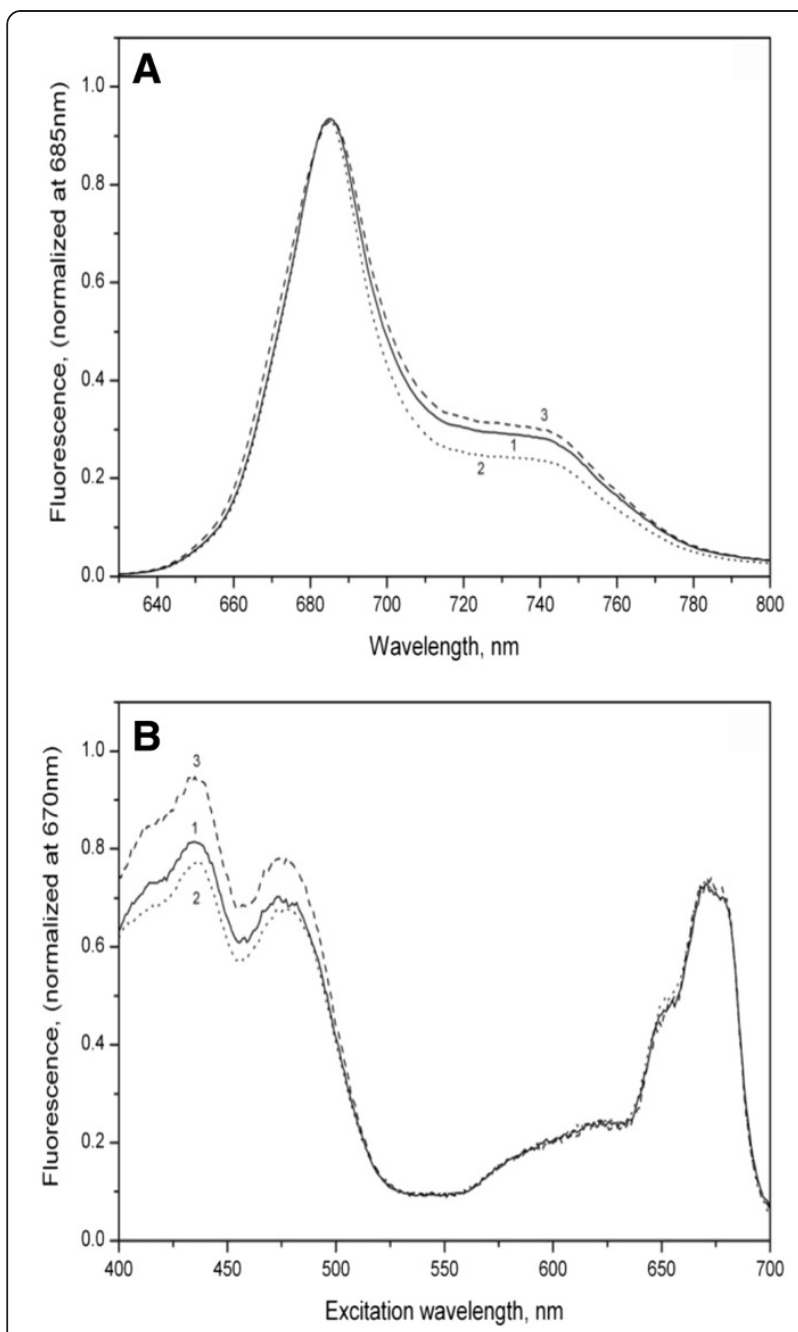

Figure 2 Fluorescence spectra. (A) under excitation at $430 \mathrm{~nm}$ (B) at $720 \mathrm{~nm}$ in wildtype (curve 1) and mutant strains Parachlorella kessleri cells (PCMut2- curve 2) and (PCMut4 -curve 3). Fluorescence spectra are normalized to the maximum fluorescence values at $690 \mathrm{~nm}$. Fluorescence excitation spectra are normalized to corresponding values of the red absorption maximum at $680 \mathrm{~nm}$.

For more detailed investigation of photosynthetic activity of wild type and two mutant strains P. kessleri cells, fluorescence parameters were measured by using M-PEA2 (Figure 4). M-PEA-2 provides extended possibilities to evaluate the functional state of PSII and PSI by measuring light-induced kinetics of the PF (OJIP transients), DF, and MR with a high resolution (Strasser et al. 2010; Oukarroum et al. 2013). Although there is no full consensus on the origin of the complex OJIP rise kinetics, it is commonly accepted that the underlying mechanisms may involve successive decrease in photochemical and non-photochemical fluorescence quenching due to the light-induced PSII closure and PQs reduction (Antal et al. 2009; Strasser et al. 2010). PSII closure refers to reduction of the primary quinone acceptor, $\mathrm{Q}_{\mathrm{A}}$, in PSII.
Figure 4 presents kinetic curves of fluorescence induction after switching the light on, normalized against the O level. In the control cells, the shape of the fluorescence curve corresponded to that described in the literature (Lazár 2009; Strasser et al. 2010). Usually, several components are observed in the kinetics of fluorescence induction in response to intense illumination, namely, the O-J-I-P transitions. The initial level $\mathrm{O}$ corresponds to chlorophyll fluorescence intensity with open RCs of PSII $\left(F_{O}\right)$ when all $\mathrm{Q}_{\mathrm{A}}$ are oxidized. The time period required to reach this level is up to $20 \mu \mathrm{s}$. The O-J phase is caused by light induced reduction of $\mathrm{Q}_{\mathrm{A}}$, while the subsequent phases reflect mainly its further accumulation in reduced state, caused by a decrease in its reoxidation rate as a result of reduction of $\mathrm{Q}_{\mathrm{B}}$ acceptors and the pool of quinone. The shape of the O-J-I-P curve was changed in the mutants (Figure 4). The mutant $P C$ Mut4 showed marked decrease in the contribution of the photochemical phase J-I-P, which indicates interruption of the electron flow from PSII to plastoquinone pool. To quantitatively analyze the characteristics of the primary processes of photosynthesis on the basis of O-J-I-P kinetic curve parameters, the so called JIP test (Strasser et al. 2010) was used. The JIP test uses the following parameters of the fluorescence induction kinetic curve: (a) fluorescence intensity at $20 \mu \mathrm{s}\left(F_{O}\right), 300 \mu \mathrm{s}(\mathrm{F} 300 \mu \mathrm{s}), 2 \mathrm{~ms}\left(F_{J}\right), 30 \mathrm{~ms}\left(F_{I}\right), 6 \mathrm{~s}$ $\left(F_{6 s}\right)$, and $F_{P}\left(F_{M}\right.$, the maximum fluorescence yield); (b) time to reach the maximum fluorescence $\left(t F_{M}\right)$, and (c) area under the kinetic curve below the $F_{M}$ level. These characteristics were used to calculate the following parameters presented in (Table 1): (1) maximum efficiency of PSII $\left(F_{V} / F_{M=}\left(F_{M}-F_{O}\right) / F_{M}\right)$; (2) relative amplitude of the O-J phase $\left(V_{J}=\left(F_{J}-F_{O}\right) / F_{V}\right.$, which reflects the fraction of non $\mathrm{Q}_{\mathrm{B}}$-reducing $\mathrm{RC}$ of PSII, which lack the contact between the two consecutive PSII acceptors, $\mathrm{Q}_{\mathrm{A}}$ and $\mathrm{Q}_{\mathrm{B}}$; (3) relative amplitude of the J-I phase $\left(V_{\mathrm{I}}=\left(F_{\mathrm{I}}-F_{\mathrm{J}}\right) / F_{V}\right)$ : (4) $M_{\mathrm{O}}$ parameter $\left(M_{\mathrm{O}}=4 \times\left(F_{300 \mu \mathrm{s}}-F_{\mathrm{O}}\right) / F_{V}\right)$, which reflects the initial slope of the induction curve; $M_{\mathrm{O}}$ value is proportional to the rate of $\mathrm{Q}_{\mathrm{A}}$ reduction under conditions when $\mathrm{Q}_{\mathrm{B}}$ and the pool of plastoquinones are mainly in the oxidized state; (5) $S_{M}=\left(\right.$ Area) $/ F_{V}$ normalized value of $F_{V}$ the area between the OJIP curve and $F_{M}$ value, which reflects the total number of PSII turnover during the OJIP phase of growth of the fluorescence yield; (6) $A B S / R C=M_{O}\left(1 / F_{M}\right)\left(l / V_{J}\right)$, average value of absorbed photon streams in RC of PSII (observed size of the active PSII antenna); (7) capacity for $\mathrm{pH}$ induced nonphotochemical fluorescence quenching $\left(q_{\mathrm{E}}=\left(F_{M}-F_{6 s}\right) /\right.$ $\left.F_{V}\right)$; and (8), capability of the quinone pool to quench fluorescence $q_{\mathrm{PQ}}=\left(F_{M}-F_{I}\right) / F_{V}$. Many parameters of the JIP-test are mutually dependent; that is, change in some of them leads to changes in the others. Independent parameters $\left(V_{\mathrm{J}}\right.$ and $\left.M_{\mathrm{O}}\right)$ derived form the analysis of the $\mathrm{O}-\mathrm{J}$ phase provide the information on $\mathrm{Q}_{\mathrm{A}}$ reduction. 

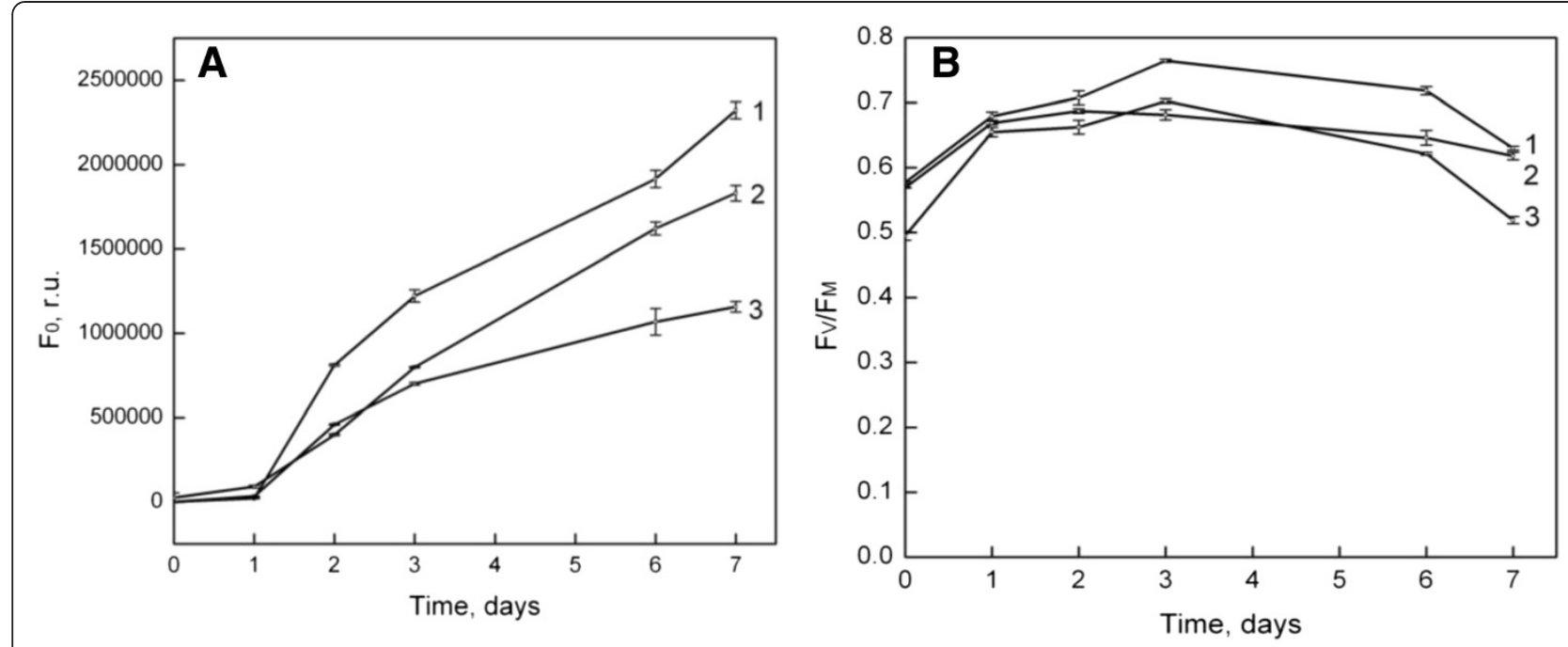

Figure 3 Changes in fluorescence parameters. (A) $F_{O}$ (B) $F_{V} / F_{M}$ in suspension wildtype (curve 1) and mutant strains of Parachlorella kessleri cells PCMut2 (curve 2) and PCMut4 (curve 3 ) during growth. The graphs are plotted as means \pm SEM.

From the $V_{\mathrm{I}}$ and $S_{\mathrm{M}}$ parameters, it is possible to derive information on further accumulation of reduced which occurs due to reduction of $\mathrm{Q}_{\mathrm{B}}$ and the quinone pool.

It is assumed that the $\mathrm{O}-\mathrm{J}$ phase of fluorescence induction reflects the accumulation of both reducing and non-reducing PSII (Antal et al. 2009; Strasser et al. 2010). The hypothesis is supported by the fact that DCMU (3-(3,4-dichlorophenyl)-1,1-dimethylurea), which inhibits electron transport between $\mathrm{Q}_{\mathrm{A}}$ and $\mathrm{Q}_{\mathrm{B}}$, leads to fast growth of fluorescence to the maximum level within $2 \mathrm{~ms}$, corresponding to the appearance of the J peak in the control. Analysis demonstrated that, in cells of the strains obtained, the number of non- $\mathrm{Q}_{\mathrm{B}}$-reducing centers of PSII, which are incapable of the quinone pool reduction, was increased. This was supported by a number of other parameters (Table 1). Accordingly, values of the parameter $M_{O}$, which reflects the initial slope of the induction curve, increased. Total flow of photons, absorbed by the pigments of the PSII antenna and normalized against the $\mathrm{RC}$ number $(A B S / R C)$, increased in the cultures of mutant strains P. kessleri, as compared to the control values. At the same time, the relative amplitude of the J-I phase $\left(\mathrm{V}_{\mathrm{I}}\right)$ was practically unchanged.

Prompt fluorescence induction curves also demonstrated the suppression of fluorescence decay after reaching the maximum due to $\Delta \mathrm{pH}$ dependent nonphotochemical quenching $\left.\left(q_{\mathrm{E}}=\left(F_{M}-F_{6 s}\right) / F_{V}\right)\right)$. The value of this parameter decreased in mutant strains, which evidences a decrease in the membrane energization. The ability of the quinone pool to quench fluorescence $q_{\mathrm{PQ}}=\left(F_{M}-F_{I}\right) / F_{V}$ in algae of mutant strains was also reduced.

Figure 5 shows the kinetics of fluorescence decay in the wild type and mutant strains of $P$. kessleri cells. Biexponential fitting of decay kinetics was applied to distinguish two components, one of them is related to energy trapping (fast component) and the other to charge stabilization and recombination in PSII reaction centers (slow ccomponent) (Volgusheva et al. 2007.).

In our experiments we found a fast component with half-time about $1 \mathrm{~ns}$ and a slower component with halftime more than 2 ns (Table 2). In the wild type and $P C$ Mut2, the ratio of these components was 40 and $60 \%$, respectively. In PC Mut4, the half-time of the slow component increased from 2.2 to $2.5 \mathrm{~ns}$, and its relative magnitude also increased to $72 \%$.

Slower fluorescence decay and an increase in the contribution of the slow component, generally, reflect a higher reduction of the PSII $Q_{A}$ acceptor. It is clearly seen in the presence of DCMU, an inhibitor of electron transport. The fact of slower fluorescence decay in PC Mut4 agrees well with the increase in the concentration of non- $\mathrm{Q}_{B}-$ reducing centers of PSII in cells of this mutant strain.

Reflectance changes at $820 \mathrm{~nm}(\mathrm{MR})$ are assigned to the redox transitions of $\mathrm{P}_{700}$, a primary electron donor in PSI, and plastocyanin, an electron donor for $\mathrm{P}_{700}{ }^{+}$. For the simplicity, further in the text we refer MR signal to $\mathrm{P}_{700}$. In the dark adapted state, $\mathrm{P}_{700}$ is in the neutral state and the reflectance at $820 \mathrm{~nm}$ is high. After the onset of illumination MR value rapidly declines, reaching a dip at about $10 \mathrm{~ms}$, followed by the slow recovery to the initial level. These changes reflect the rapid oxidation of $\mathrm{P}_{700}$ by the acceptor side of PSI, and the subsequent reduction of $\mathrm{P}_{700}{ }^{+}$by electrons arriving from PSII (Figure 4B) shows MR transients normalized to the initial value at $20 \mu \mathrm{s}\left(\mathrm{MR} / \mathrm{MR}_{0}\right)$ recorded simultaneously with the OJIP curves described above.

In $P C$ Mut 2 mutant strain, the initial oxidation, as well as reduction of $\mathrm{P}_{700}$ was similar to those in the wild type. 


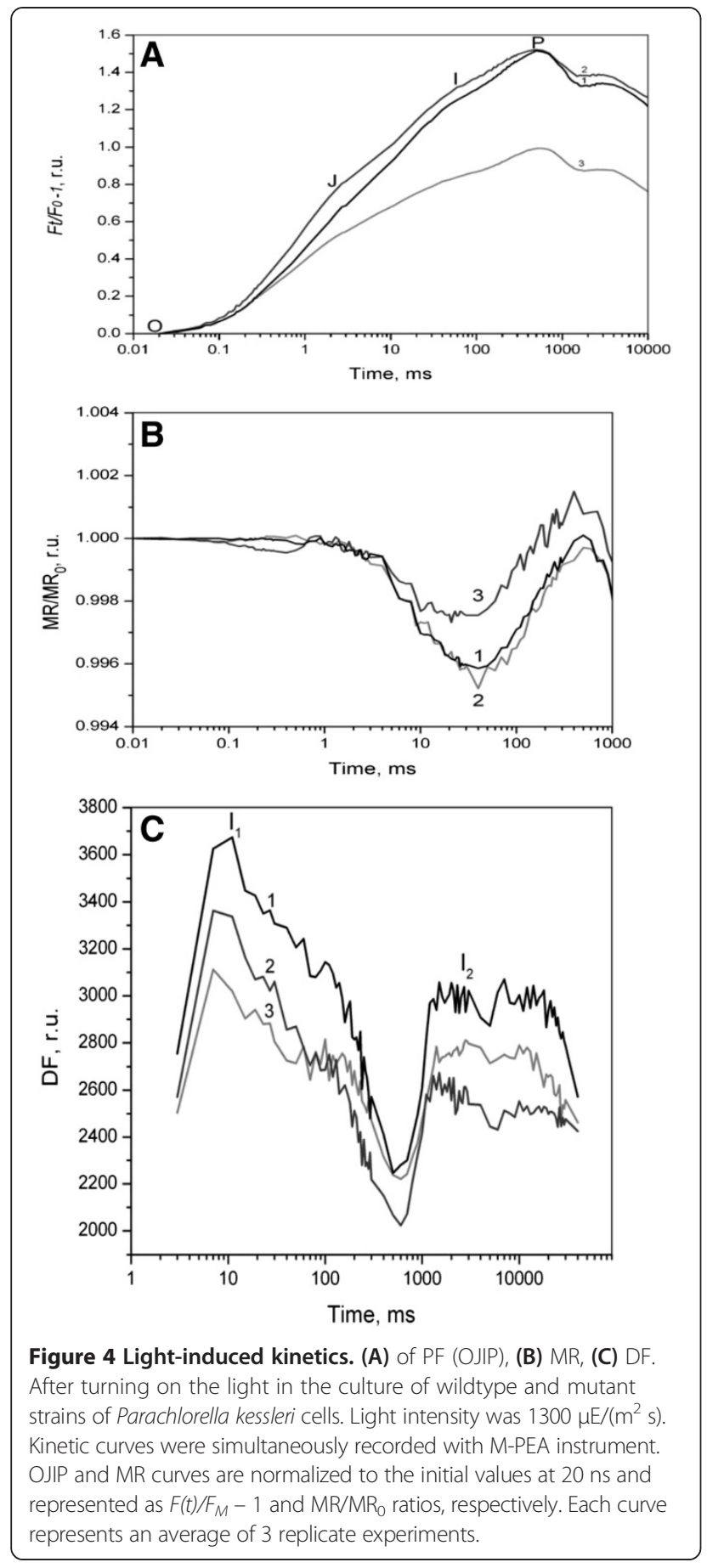

In PC Mut4 mutant strain, $\mathrm{P}_{700}$ oxidation was reduced, indicating an inhibition of electron transport via PSI.

Both DF and PF emission originates from radiative deactivation of the singlet excited state of antennae $\mathrm{Chl}$ in PSII (Goltsev et al. 2009). In the case of PF, excited states are created mainly through the absorption of photons by photosynthetic pigments, whereas DF emission is coupled to excited states formed as a result of the backward reactions of electron transport. In order to separate a low yield DF emission from high yield PF signal, DF is recorded in the dark after the excitation pulse offset. DF intensity is proportional to the rates of the corresponding recombination reactions which are strongly affected by the energization of a thylakoid membrane (Wraight and Crofts 1971). Thus, changes of DF yield during the induction period depend on the formation of electrical and proton differences across the membrane. Fast and slow phases of the DF induction kinetics recorded with M-PEA in plant leaves have recently been analyzed (Goltsev et al. 2009; Oukarroum et al. 2013). It is assumed that the fast component $\left(\mathrm{I}_{1}\right)(\sim 10-100 \mathrm{~ms})$ reflects transient formation of the electrical gradient across the membrane, likely, due to generation of charge pairs in PSI $\left(\mathrm{P}_{700}^{+}[\mathrm{Fe}-\mathrm{S}]^{-}\right)$. The presence of the second DF peak, $\mathrm{I}_{2}$, in the seconds range, is associated with the light induced formation of the proton transmembrane gradient $(\Delta \mathrm{pH})$ that increases the constant of rate of emission transitions in the RC of PSII.

Peaks on DF curve at $20-50 \mathrm{~ms}$, and $1 \mathrm{~s}$ were reduced in mutant strains, indicating a decrease in the formation of electrical and proton potential differences across the thylakoid membrane. However, the fast component of DF was reduced to a greater extent in PC Mut4 strain. The slow phase of the DF induction kinetics $(\Delta \mathrm{pH})$ was reduced more significantly in $P C M u t 2$, compared to the wild type. The last fact agrees with the reduced non-photochemical quenching $q_{\mathrm{E}}=\left(F_{M}-F_{6 s}\right) / F_{V}$ in $P C$ Mut2 (Table 1).

These data were confirmed by measurement of fluorescence parameters in algae with a Water-PAM pulse fluorometer. The relative rate of the noncyclic electron transport (ETR), calculated as $r E T R=Y \times E_{i} \times 0.5$, and nonphotochemical fluorescence quenching $N P Q=\left(F_{M}-F_{M}{ }^{\prime}\right) /$ $F_{M}$ ' by electrochemical gradient of protons were reduced in mutants (Table 3).

Thus, simultaneous registration of the kinetics of prompt, delayed fluorescence and redox state of $\mathrm{P}_{700}$ made it possible to monitor the individual reactions of accumulation of reduced carriers between the photosystems and kinetics of electrochemical proton gradient on a thylakoid membrane of mutant strains $P$. kessleri.

It is known that the constant fluorescence level $F_{O}$ highly correlates with the total content of pigments in the photosynthetic apparatus of algal cells that harvest light energy (Matorin et al. 2004). The chlorophyll $a$ and $b$ content in the wild type were $53.3 \pm 5.7,30.2 \pm 2.4 \mathrm{mg} / \mathrm{l}$ respectively while in the $P C M u t 2$ were $48.3 \pm 2.3,25.2 \pm$ $2.3 \mathrm{mg} / \mathrm{l}$ respectively and in the PCMut4 were $33.3 \pm 3.2$, $10.2 \pm 3.6 \mathrm{mg} / \mathrm{l}$ respectively. Fluorescence parameters are used long ago in hydrobiology and oceanology for an assessment of the concentration of microalgae in water and their photosynthetic activity. There is a large number various the submersible fluorometers of different firms for these purposes. Researchers have therefore been trying 
Table 1 Parameters of OJIP kinetics of fluorescence induction curve of wild type and three mutant strains Parachlorella kessleri cells

\begin{tabular}{|c|c|c|c|c|}
\hline \multicolumn{2}{|c|}{ JIP-test parameters } & \multirow{2}{*}{$\begin{array}{l}\text { Wild type } P C^{*} \\
0.610 \pm 0.01\end{array}$} & \multirow{2}{*}{$\frac{P C \text { Mut2* }}{0.607 \pm 0.01}$} & \multirow{2}{*}{$\frac{\text { PC Mut4* }}{0.506 \pm 0.01}$} \\
\hline$F_{V} / F_{M}$ & Maximum quantum yield of charge separation in PS2 & & & \\
\hline$V_{\jmath}$ & Relative amplitude of the O-J phase & $0.38 \pm 0.1$ & $0.47 \pm 0.01$ & $0.47 \pm 0.003$ \\
\hline$V_{1}$ & Relative amplitude of the $\mathrm{J}$-I phase & $0.96 \pm 0.02$ & $0.96 \pm 0.01$ & $0.94 \pm 0.004$ \\
\hline Mo & Initial slope of the O-J fluorescence phase & $0.60 \pm 0.04$ & $0.73 \pm 0.02$ & $0.87 \pm 0.03$ \\
\hline$S_{M}$ & $\begin{array}{l}\text { Area between the fluorescence kinetic curve }(\mathrm{O}-J-I-P) \text { and the } \\
\text { level of Fm normalized against the Fv value }\end{array}$ & $40.97 \pm 4.24$ & $38.72 \pm 2.32$ & $30.06 \pm 1.01$ \\
\hline$A B S / R C$ & $\begin{array}{l}\text { Average value of absorbed photon flows in PS2 RC (of apparent } \\
\text { size of the active antenna in PS2) }\end{array}$ & $2.57 \pm 0.2$ & $2.85 \pm 0.1$ & $3.51 \pm 0.2$ \\
\hline$q E$ & Capacity for pH-induced non-photochemical fluorescence quenching & $0.14 \pm 0.01$ & $0.12 \pm 0.01$ & $0.10 \pm 0.01$ \\
\hline$q P Q$ & Capacity of the quinone pool for fluorescence quenching & $0.24 \pm 0.04$ & $0.20 \pm 0.01$ & $0.19 \pm 0.02$ \\
\hline
\end{tabular}

*Figures are expressed as mean $\pm \mathrm{SD}$.

The kinetic parameters of fluorescence induction were recorded with M-PEA-2 instrument under actinic illumination of $1300 \mu$ mol photons $\mathrm{m}^{-2} \mathrm{~s}^{-1}$.

to find a method of determining the chlorophyll $a$ concentration from in situ fluorescence measurements. These would cover not only fluorescence induced naturally by sunlight but also that induced by artificial light sources. Measurements of the latter are either contact measurements carried out in situ with submersible fluorometers or remote methods using lidars (Pogosyan and Matorin 2005).

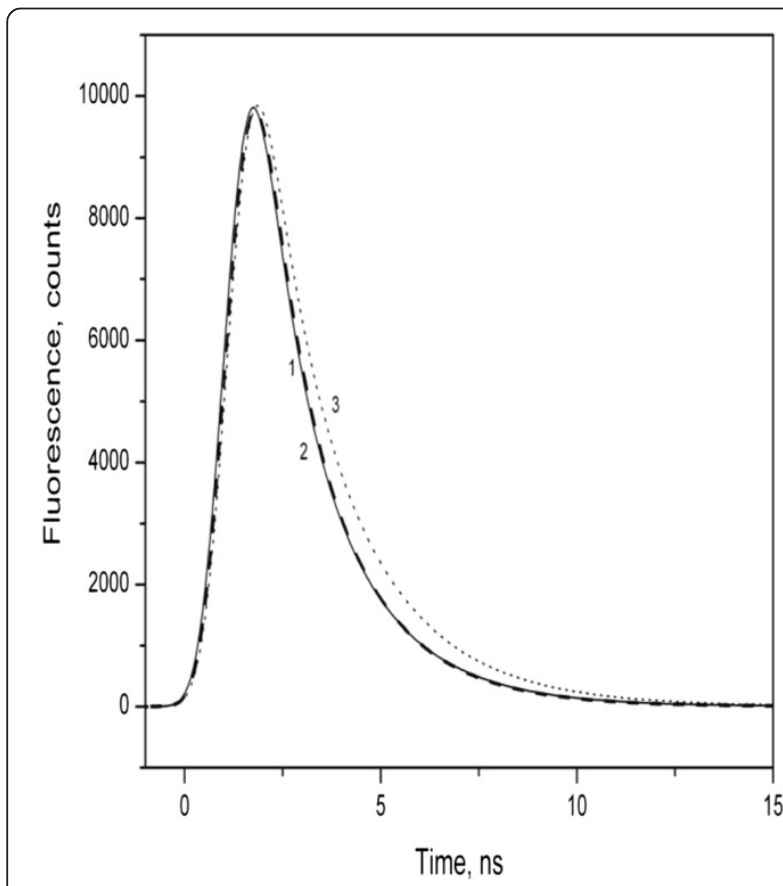

Figure $\mathbf{5}$ Kinetics of fluorescence decay. In wildtype (curve 1) and mutant strains of Parachlorella kessleri cells (PCMut2-curve 2) and (PCMut4 -curve 3). The measurements were performed with a Fluorolog-3 spectrofluorometer at $685 \mathrm{~nm}$, using TCSPC method with sample activation with light at $1 \mathrm{Mhz}$ frequency with a LED $390 \mathrm{~nm}$ by pulses with half-widths of $0.9 \mathrm{~ns}$.

\section{Conclusion}

Microalgae, which use sun energy for synthesis of organic matter, are the main energy source in aquatic systems and serve as the food for other organisms. The linear chain of photosynthetic electron transport comprises two sequentially operating photosystems (PSII and PSI) interconnected through intermediary quinone pool of electron carriers. According to the Z-scheme of electrontransport chain (ETC.), activities of each photosystem affect the redox state of the other system. Such a relation between PSII and PSI is evident from chlorophyll fluorescence, whose level depends on the redox state of the quinone acceptor $\mathrm{Q}_{\mathrm{A}}$, as well as from the redox state of chlorophyll $\mathrm{P}_{700}$, the primary electron donor of PSI. The photoreaction of PSII reduces $\mathrm{Q}_{\mathrm{A}}$, thus raising chlorophyll fluorescence and the extent of $\mathrm{P}_{700}$ reduction, while the PSI reaction converts $\mathrm{P}_{700}$ into its oxidized form $\left(\mathrm{P}_{700}{ }^{+}\right)$, which promotes $\mathrm{Q}_{\mathrm{A}}$ oxidation and fluorescence lowering. Simultaneous chlorophyll fluorescence and $820 \mathrm{~nm}$ transmission measurements by using M-PEA-2 allow evaluating the functional state of PSII and PSI. Analysis of fluorescence induction curves demonstrated inhibition of electron transport in PSII and an increase fraction of non- $\mathrm{Q}_{\mathrm{B}}$-reducing centers. This manifested itself also in an increase of the slow component in decay curve of fluorescence with half-time 2 ns in $P C$ Mut4. In addition, analysis of induction curves of the

Table 2 Changes of amplitude and duration of fast (A1, $\left.\tau_{1}\right)$ and slow $\left(A 2, \tau_{2}\right)$ components of fluorescence decay kinetics of wild type and mutant strains Parachlorella kessleri cells

\begin{tabular}{lllll}
\hline Samples & A1 (\%) & A2 (\%) & $\boldsymbol{T}_{\mathbf{1}}$ (ns) & $\mathbf{T}_{\mathbf{2}}$ (ns) \\
\hline Wild type & 40 & 60 & $1.0 \pm 0.13$ & $2.24 \pm 0.03$ \\
PC Mut 2 & 37 & 63 & $0.97 \pm 0.14$ & $2.18 \pm 0.03$ \\
PC Mut 4 & 28 & 72 & $1.09 \pm 0.23$ & $2.47 \pm 0.04$ \\
\hline
\end{tabular}

*Figures are expressed as mean \pm SD. 
Table 3 Changes of fluorescence parameters of wild type and mutant strains of Parachlorella kessleri cells

\begin{tabular}{llll}
\hline Fluorescence parameters & Wild type & PC Mut2 & PC Mut \\
\hline$F_{V} / F_{M}$ & \\
NPQ & $0.58 \pm 0.01$ & $0.5 \pm 0.01$ & $0.45 \pm 0.02$ \\
$r E T R_{\text {max }}$ (r.u.) & $0.179 \pm 0.02$ & $0.133 \pm 0.003$ & $0.083 \pm 0.004$ \\
\hline
\end{tabular}

*Figures are expressed as mean $\pm S D$.

$F_{V} / F_{M}$ - samples in darkness, $r E T R_{\max }$ - maximum relative rate of electron transport and $N P Q=\left(F_{M}-F_{M}{ }^{\prime}\right) / F_{M^{\prime}}-$ non-photochemical fluorescence quenching at illumination $800 \mu \mathrm{mol}$ photons $\mathrm{m}^{-2} \mathrm{~s}^{-1}$.

delayed fluorescence and a decrease of NPQ revealed a decrease in the energization of photosynthetic membranes in mutant cells. Our study showed that the most sensitive parameters are induction curves of prompt and delayed fluorescence in mutant algal cells. Selected parameters are indeed viable tools for diagnostic alga state in biotechnological studies during obtaining mutant forms.

\section{Abbreviations}

PSII: Photosystem II; RC: Reaction center; $Q_{A}$ : Primary; $Q_{B}$ : Secondary quinone acceptors of electron; PQ: Plastoquinone; O: J, I, P, Intermediate levels of the light-induced fluorescence curve; DF: Delayed Chl fluorescence;

$P_{700}$ : Pigment of the RC of PSI.

\section{Competing interest}

The authors declare that they have no competing interest.

\section{Authors' contribution}

All authors read and approved the final manuscript.

\section{Acknowledgement}

The authors acknowledge department of biotechnology, Al-Farabi Kazakh National University which granted materials used in this research and department of biophysics, Moscow State University for supporting material analysis.

\section{Author details}

${ }^{1}$ Department of Biotechnology, Al-Farabi Kazakh National University, 71, Al-Farabi, ave, Almaty 050040, Kazakhstan. ${ }^{2}$ Faculty of Biology, Moscow State University, Vorobyevi Gory 119992, Moscow, Russia. ${ }^{3}$ Botany Department, Faculty of Science, Suez Canal University, Ismaillia 41522, Egypt.

Received: 14 April 2014 Accepted: 23 June 2014

Published online: 12 July 2014

\section{References}

Antal TK, Matorin DN, llyash LV, Volgusheva AA, Osipov VA, Konyuhov IV, Krendeleva TE, Rubin AB (2009) Probing of photosynthetic reactions in four phytoplanktonic algae with a PEA fluorometer. Photosynth Res 102:67-76

Bulychev AA, Osipov VA, Matorin DN, Vredenberg WJ (2013) Effects of far-red light on fluorescence induction in infiltrated pea leaves under diminished $\Delta \mathrm{pH}$ and $\Delta \varphi$ components of the proton motive force. J Bioenerg Biomembr 45:37-45

Charles DA (2008) Algal Chemical Ecology.ch. 13, U. Springer-Verlag Berlin Heidelberg, Karsten

Fott B, Nováková M (1969) A monograph of the genus Chlorella. The fresh-water species. In: Fott B (ed) Studies in Phycology. Academia, Prague, pp 10-74

Goltsev V, Zaharieva I, Chernev P, Strasser RJ (2009) Delayed fluorescence in photosynthesis. Photosynth Res 101:217-232

Gorman DS, Levine RP (1965) Proc Natl Acad Sci U S A 54:1665-1669

Harris EH (1989) The Chlamydomonas Sourcebook: A Comprehensive Guide to Biology and Laboratory Use. Academic Press, San Diego, 780

Herlory O, Richard P, Blanchard GF (2007) Methodology of light response curves: Application of chlorophyll fluorescence to microphytobenthic biofilms. Mar Biol 153:91-101

Holzinger A, Lütz C (2006) Algae and UV irradiation: effects on ultrastructure and related metabolic functions. Micron 35:190-207
Krienitz L, Hegewald EH, Hepperle D, Huss VAR, Rohr T, Wolf M (2004) Phylogenetic relationship of Chlorella and Parachlorella gen. nov. (Chlorophyta, Trebouxiophyceae). Phycologia 43:529-42

Lazár D (2009) Modelling of light-induced chlorophyll a fluorescence rise (O-J-I-P transient) and changes in $820 \mathrm{~nm}$-transmittance signal of photosynthesis. Photosynthetica 47:483-498

Lippemeier S, Harting P, Colijn F (1999) Direct impact of silicate on the photosynthetic performance of the diatom Thalassiosira weissflogii assessed by on and off-line PAM fluorescence measurements. J Plankton Res 21:269-283

Matorin DN, Rubin AB (2012) Chlorophylla fluorescence in higher plants and algae Moscow. IKI-RKhD, Izhevsk, p 256

Matorin DN, Antal TK, Ostrowska M, Rubin AB, Ficek D (2004) Chlorophyll fluorometry as a method for studying light absorption by photosynthetic pigments in marine algae. Oceanologia 46:519-531

Matorin DN, Todorenko DA, Seifullina NK, Zayadan BK, Rubin AB (2013) Effect of silver nanoparticles on the parameters of chlorophyll fluorescence and P700 reaction in the green alga Chlamydomonas reinhardtii. Microbiology 82:862-867

Merchant SS, Simon EP, Olivier V (2007) The Chlamydomonas Genome Reveals the Evolution of Key Animal and Plant Functions. Sci 318:245-251

O'Connor DV, Phillips D (1984) Time correlated single photon counting. Academic Press, London

Oukarroum A, Goltsev V, Strasser RJ (2013) Temperature effects on pea plants probed by simultaneous measurements of the kinetics of prompt fluorescence, delayed fluorescence and modulated $820 \mathrm{~nm}$ reflection. PLoS One 8:1-10

Pogosyan SI, Matorin DN (2005) Variability in the state of the photosynthetic system of the Black Sea phytoplankton. Oceanology 45(1):139-148

Schansker G, To'th SZ, Holzwarth AR, Garab G (2014) Chlorophyll a fluorescence: beyong the limits of the Qa model. Photosynth Res 120:43-58

Schreiber U (2004) Pulse-Amplitude (PAM) fluorometry and saturation pulse method: an overview. In: Papageorgiou G, Govindjee (eds) Chlorophyll fluorescence: A signature of Photosynthesis and Respiration, vol 9. Springer, Dordrecht, pp 79-319

Strasser RJ, Tsimilli-Michael M, Srivastava A (2005) Analysis of chlorophyll a fluorescence transient. In: Papageorgiou GC and Govindjee (eds) Chlorophyll fluorescence: a signature of photosynthesis. Kluwer Academic Publishers, Dordrecht/Boston/London, pp 321-362

Strasser RJ, Tsimilli-Michael M, Sheng Q, Goltsev V (2010) Simultaneous in vivo recording of prompt and delayed fluorescence and 820-nm reflection changes during drying and after rehydration of the resurrection plant Haberlea rhodopensis. Biochim Biophys Acta 1797:1313-1326

Volgusheva AA, Zagidullin VE, Antal TK, Korvatovsky BN, Krendeleva TE, Paschenko VZ, Rubin AB (2007) Examination of chlorophyll fluorescence decay kinetics in sulfur deprived algae Chlamydomonas reinhardtii. Biochim Biophys Acta 1767:559-564

Wraight CA, Crofts AT (1971) Delayed fluorescence and the high-energy state of chloroplasts. Eur J Biochem 19:386-397

doi:10.1186/s40529-014-0053-7

Cite this article as: Saleh et al:: Differentiation between two strains of microalga Parachlorella kessleri using modern spectroscopic method. Botanical Studies 2014 55:53.

\section{Submit your manuscript to a SpringerOpen ${ }^{\circ}$ journal and benefit from:}

- Convenient online submission

- Rigorous peer review

- Immediate publication on acceptance

- Open access: articles freely available online

- High visibility within the field

- Retaining the copyright to your article

Submit your next manuscript at $>$ springeropen.com 